\title{
The Efficacy of Neurofeedback Among Patients with Major Depressive Disorder: Preliminary Study
}

\author{
San-Yu Wang ${ }^{1}$, I-Mei Lin ${ }^{1^{*}}$, Erik Peper ${ }^{2}$, Yu-Ting Chen ${ }^{1}$, Tze-Chun Tang ${ }^{3,4}$, Yi-Chun Yeh ${ }^{3}, Y u-$ \\ Che Tsai ${ }^{1}$, and Che-Cheng $\mathrm{Chu}^{1}$

\begin{abstract}
${ }^{1}$ Department of Psychology, College of Humanities and Social Sciences, Kaohsiung Medical University, Taiwan
${ }^{2}$ Institute for Holistic Health Studies, San Francisco State University, California, USA

${ }^{3}$ Department of Psychiatry, Kaohsiung Medical University Hospital, Taiwan

${ }^{4}$ Dr. Tang's Psychiatric Clinic and Mind Center, Taiwan
\end{abstract}

\begin{abstract}
Introduction: Alpha asymmetry of the left and right frontal hemisphere is a potential biomarker for major depressive disorder (MDD). Neurofeedback (NFB) is a clinical intervention program for regulating brain activity and decreasing alpha asymmetry. The purpose of this study was to explore the efficacy of NFB among patients with MDD. Methods: Fourteen patients with MDD were randomly assigned to a NFB group that received neurofeedback training $1 \mathrm{hr}$ weekly for 6 weeks and to a control group that was treated without training. A 5-min resting baseline of electroencephalogram (EEG) was recorded at F3 (left) and F4 (right) before and after NFB, and the alpha power was analyzed as an asymmetry index (A1). Results: The A1 of the control group decreased from pre- to post-interventions while the A1 of the NFB group increased from pre- to post-interventions. Anxiety and depression scores of the responder group decreased from pre- to post-interventions, while the scores of the non-responder group increased from pre- to post-interventions. Conclusion: Patients who respond to the NFB training showed a decrease in anxiety and depression scores compared to those who do not. This study indicated that NFB could improve left frontal hypoarousal or right frontal hyperarousal among patients with MDD.
\end{abstract}

Keywords: major depressive disorder; neurofeedback; electroencephalogram; alpha asymmetry

Citation: Wang, S.-Y., Lin, I.-M., Peper, E., Chen, Y.-T., Tang, T.-C., Yeh, Y.-C., .... Chu, C.-C. (2016). The efficacy of neurofeedback among patients with major depressive disorder: Preliminary study. NeuroRegulation, 3(3), 127-134. http://dx.doi.org/10.15540/nr.3.3.127

*Address correspondence to: I-Mei Lin, PhD, 100, Shih-Chuan 1st Edited by:

Road, Kaohsiung, 80708, Taiwan. Email: psyiml@kmu.edu.tw

Rex L. Cannon, PhD, Neurogifted, Boynton Beach, Florida, USA

Copyright: (c) 2016. Wang et al. This is an Open Access article distributed under the terms of the Creative Commons Attribution License (CC-BY).

Reviewed by:

Rex L. Cannon, PhD, Neurogifted, Boynton Beach, Florida, USA Ed Pigott, PhD, Positive Brain Training, Wellington, FL, USA

\section{Introduction}

Major depressive disorder (MDD) is one of the common mental disorders that cause individual physical discomfort and emotional suffering, as well as interpersonal problems, and impaired social and career functioning. Depression is usually treated with antidepressant medications; however, recent research suggests that for children and teenagers with major depression, 13 out of the 14 antidepressant drugs do not work and can increase teenage suicide rates (Le Noury et al., 2015). Even in adults, selective serotonin reuptake inhibitors, such as Prozac, may not work any better than placebo; in addition, almost all studies that have demonstrated positive pharmaceutical effects have been directly or indirectly sponsored by pharmaceutical companies (Ebrahim, Bance, Athale, Malachowski, \& Ioannidis, 2016). To avoid antidepressant side effects and offer patients MDD treatment, neurofeedback (NFB) is a promising new approach.

Electroencephalographic (EEG) studies have found greater alpha power over the left than the right frontal regions among patients with MDD (Debener et al., 2000; Gotlib, Ranganath, \& Rosenfeld, 1998). Davidson (1984) indicated that the frontal alpha asymmetry with an active left frontal lobe is related to the behavioral approach system (BAS), leading to more positive emotions, motivation, and behavioral approaches. On the other hand, an active right prefrontal lobe is related to behavioral inhibition system (BIS), leading to more negative emotions, 
escape-related motivation, and behavioral withdrawal.

Davidson (1998) calculated the alpha asymmetry score (A score) from the alpha power at the left frontal ( $L$ or $F 3$ ) and right frontal ( $R$ or $F 4$ ) lobes. Baehr, Rosenfeld, and Baehr proposed two equations for the $A$ score: $A 1=\log (R)-\log (L)$ and $A 2=(R-L) /(R+L)$ (Baehr, Rosenfeld, \& Baehr, 1997; Rosenfeld, Baehr, Baehr, Gotlib, \& Ranganath, 1996). When the alpha power at the right frontal lobe is higher than that in the left frontal lobe, the A1 score is positive and is associated with more positive emotions (such as happiness and joy), motivation, and approach behavior from the BAS system. When the alpha power at the right frontal lobe is lower than that in the left frontal lobe, the A1 score is negative and is associated with more negative emotions (such as fear, disgust, and sadness), escape-related motivation, and behavior withdrawal from the BIS system (Davidson, 1984). The prefrontal alpha asymmetry suggests brain lateralization (hemispheric laterality; Davidson, 1984, 1993, 1998).

Patients with MDD show lower alpha asymmetry scores as compared to healthy adults (Cantisani et al., 2015; Coan and Allen, 2004; Debener et al., 2000; Kemp et al., 2010). Similar alpha asymmetry was also observed in patients with remission from MDD (Stewart, Coan, Towers, \& Allen, 2011).

Based on the concept that alpha asymmetry is a potential biomarker for depression, Baehr et al. (1997) developed a neurofeedback protocol, the Alpha Asymmetry (ALAY) protocol, to train patients to change the frontal alpha asymmetry as neurofeedback training for depression. The goals were to decrease the left frontal alpha power at F3 to improve positive emotion and increase the right frontal alpha power at F4 to decrease depression. Case reports found that the ALAY protocol could improve the frontal alpha asymmetry and decrease depressive symptoms (Baehr et al., 1997; Dias \& van Deusen, 2011; Rosenfeld et al., 1996). The treatment effectiveness was maintained through 1to 5-year follow-ups (Baehr, Rosenfeld, \& Baehr, 2001).

Several studies have shown that neurofeedback has long-term benefits and show significant improvement in the frontal alpha asymmetry and depressive symptoms. Choi et al. (2011) conducted a randomized sham control group study comparing a 1-hr twice a week for 10 weeks NFB group (ALAY protocol) with a psychotherapy group. The NFB group as compared to the psychotherapy group showed higher A1 scores, increased positive autonomic thoughts, decreased negative autonomic thoughts, and improved performance of the executive function tests, such as semantic and phonological fluencies. Peeters, Oehlen, Ronner, van Os, and Lousberg (2014) confirmed that the neurofeedback with the ALAY protocol did decrease the depression score, but did not decrease the A1 score after 10 sessions for decreasing left frontal alpha power.

Previous studies have found that the average number of sessions of the NFB protocol were between 10 and 36 sessions (Baehr et al., 1997, 2001; Choi et al., 2011; Peeters et al., 2014; Rosenfeld et al., 1996). The average treatment sessions to complete the NFB protocol are 20-22 sessions (Hammond, 2005). After 3-6 sessions of 30-min each of NFB, patients may feel the difference between pre- and post-interventions. After 10-12 sessions, patients feel a significant improvement. For sustained, long-term changes and clinical benefits of neurofeedback, 30 to 60 sessions may be required, which depends on compliance and motivation (Linden, Habib, \& Radojevic, 1996). Hammond (2005) indicated that patients might feel the differences after 3-6 sessions of neurofeedback; however, this has not been systematically explored. The purpose of this study was to examine: (1) a short-term six-session ALAY protocol of NFB among patients with MDD, and (2) the efficacy of the ALAY protocol in increasing the alpha asymmetry score and decreasing depressive symptoms among patients with MDD in Taiwan.

\section{Methods}

\section{Participants}

Fourteen patients with MDD were referred by psychiatrists based on the criteria of Diagnostic and Statistical Manual of Mental Disorders, fifth edition (DSM-5) at an outpatient clinic of Kaohsiung Medical University Hospital (American Psychiatric Association, 2013). Patients with MDD with serious physical and mental disorders (e.g., cancer, heart disease, kidney disease, Alzheimer's disease, Parkinson's disease, schizophrenia, bipolar disorder, substance abuse, etc.) were excluded from this study. The institutional review board approval was obtained from the ethics committee of the Kaohsiung Medical University Hospital (KMUH-IRB-20110063), and written informed consent was obtained from each participant before the experiment.

All participants were assigned randomly to the NFB group and the control group. There was no 
significant difference between the two groups in age, sex, Beck Anxiety Inventory (BAI), Beck Depression Inventory-II (BDI-II), and duration of disorder. Participants in both groups received medications by psychiatrists; however, there was no group difference in the categories of medications (Table 1). All participants in both groups received neither psychotherapy nor other nonpharmacological treatment at the same time. The equal group design was confirmed in this study. Participants in the NFB group received $1 \mathrm{hr}$ per week for 6 weeks of neurofeedback that was based on the ALAY protocol (Baehr et al., 1997). The ALAY protocol included down-training of the alpha power $(8-12 \mathrm{~Hz})$ at F3 and up-training of the alpha power $(8-12 \mathrm{~Hz})$ at F4, and the treatment goal was to increase the A1 score (Baehr et al., 1997; Baehr, Rosenfeld, Baehr, \& Earnest, 1998). Participants in the control group received the pharmacological treatment only.

\section{Table 1}

The Demographic Characteristics of All Participants

\begin{tabular}{lccc} 
Variable & $\begin{array}{c}\text { NFB group } \\
(n=7)\end{array}$ & $\begin{array}{c}\text { Control group } \\
(n=7)\end{array}$ & $t / \chi^{2}$ \\
\hline $\begin{array}{l}\text { Age (years) } \\
\text { Sex }\end{array} \quad$ W9.86(3.98) & $47.43(13.84)$ & -0.45 \\
$\quad$ Women & 5 & 6 & \\
$\quad$ Men & 2 & 1 & 0.42 \\
Beck Anxiety Inventory & $17.86(10.51)$ & $16.00(9.92)$ & -0.34 \\
Beck Depression Inventory-II & $30.14(10.25)$ & $22.86(13.03)$ & -1.16 \\
Duration of disorder (years) & $8.83(2.71)$ & $6.68(5.37)$ & -0.88 \\
Medications & & & \\
$\quad$ Benzodiazepine & 6 & 5 & 0.42 \\
$\quad$ Selective serotonin reuptake inhibitors & 1 & 2 & 0.42 \\
$\quad$ Atypical antidepressants & 5 & 4 & 0.31 \\
$\quad$ Sedative-hypnotic & 4 & 2 & 1.17 \\
\hline
\end{tabular}

\section{Psychological questionnaires}

All participants completed self-report questionnaires that covered demographic characteristics (such as age, sex, and years of disorder), BAI, and BDI-II at pre- and post- interventions of neurofeedback. The $\mathrm{BAI}$ and BDI-II are self-report questionnaires that measure the symptoms of anxiety and depression disorder over the course of a period, respectively.

\section{EEG equipment and measurement}

The BrainAvatar Version 4.0 (BrainMaster Technologies, Inc., Bedford, OH) EEG system was used for EEG data collection, with a 19-channel EEG cap which was placed on the participants' scalps, and linked-ear was the reference to collect EEG raw signals. All participants were measured for a 5-min baseline with eyes closed at pre- and postNFB intervention. The BioGraph Infiniti Version 6.1.1 (Thought Technology Ltd., Montreal, QC,
Canada) was used with a bipolar-channel at F3 and F4 based on the International 10-20 system, Cz was the reference, and the left earlobe served as ground (Baehr et al., 1997). The impedances of the electrode were set below $5 \mathrm{k} \Omega$; band-pass was 1-30 $\mathrm{Hz}$, notch filter was at $60 \mathrm{~Hz}$, and the sampling rate was $256 \mathrm{~Hz}$.

\section{Data reduction and statistical analysis}

The EEG raw signal was analyzed using MATLAB R2008a (The Math Works, Natick, MA), and the EEG power spectrum was transformed to the alpha power $(8-12 \mathrm{~Hz})$ by EEG insight software (Delorme \& Makeig, 2004). The frontal alpha asymmetry score (A1 index) was computed with the natural logarithms (In) by subtracting the left alpha power from the right alpha power, $A 1=\log (R)-\log (L)$ (Baehr et al., 1997). 
The $t$-tests and $\chi^{2}$ test were used to examine the equal group design on the demographic characteristics. The paired $t$-tests were used to examine the differences in the $\mathrm{A} 1$ score, $\mathrm{BAI}$, and BDI-II at pre- and post-intervention for the NFB group and control group. In addition, the changescore of $A 1=(A 1$ at post-intervention $-A 1$ at preintervention) was used to separate participants in the NFB group and control group into a responder group and non-responder group. Participants in the responder group showed an increased A1 score; the non-responders had no change in the A1 score. In addition, this study also examined the differences in the A1 score, BAI, and BDI-II at pre- and postinterventions between the responder group and the non-responder group.

\section{Results}

The treatment effectiveness of neurofeedback on the A1 score

The A1 score was increased slightly in the NFB group from pre-intervention $(M=0.11, S D=0.13)$ to post-intervention $(M=0.12, S D=0.13)$; the $A 1$ score was decreased slightly in the control group from pre-intervention $(M=0.04, S D=0.07)$ to postintervention $(M=0.02, S D=0.07$; Table 2). Although an overall increase in the alpha power at F3 and F4 was observed from pre- to postintervention in both NFB and control groups. However, there was no significant difference between the two groups in the A1 score.

\section{Table 2}

The Changes in the Alpha Power from Pre- to Post-interventions in the NFB Group and the Control Group

\begin{tabular}{crrr}
\hline Alpha Power & Pre-intervention & Post-intervention & $t$ \\
\hline NFB group $(\boldsymbol{n}=\mathbf{7})$ & & & -1.07 \\
F3 & $10.10(4.58)$ & $12.77(5.23)$ & -1.26 \\
F4 & $10.91(4.27)$ & $14.42(5.75)$ & -0.24 \\
A1 & $0.11(0.13)$ & $0.12(0.13)$ & -1.18 \\
Control group $(\boldsymbol{n}=\mathbf{7})$ & & & -1.24 \\
F3 & $5.51(3.95)$ & $7.27(5.42)$ & 0.63 \\
F4 & $5.80(4.14)$ & $7.57(5.76)$ & $0.02(0.07)$ \\
A1 & $0.04(0.07)$ & & \\
\hline
\end{tabular}

The responders showed an increased A1 score as compared to the non-responders in the NFB group.

This study used the change-score of $A 1$ to separate participants in the NFB group into a responder group and a non-responder group. Although the A1 score was not different between the two groups, four participants $(57.14 \%)$ showed increased A1 score from pre- to post-interventions; however, three of them $(42.86 \%)$ showed decreased A1 score after neurofeedback training (Figure 1). 


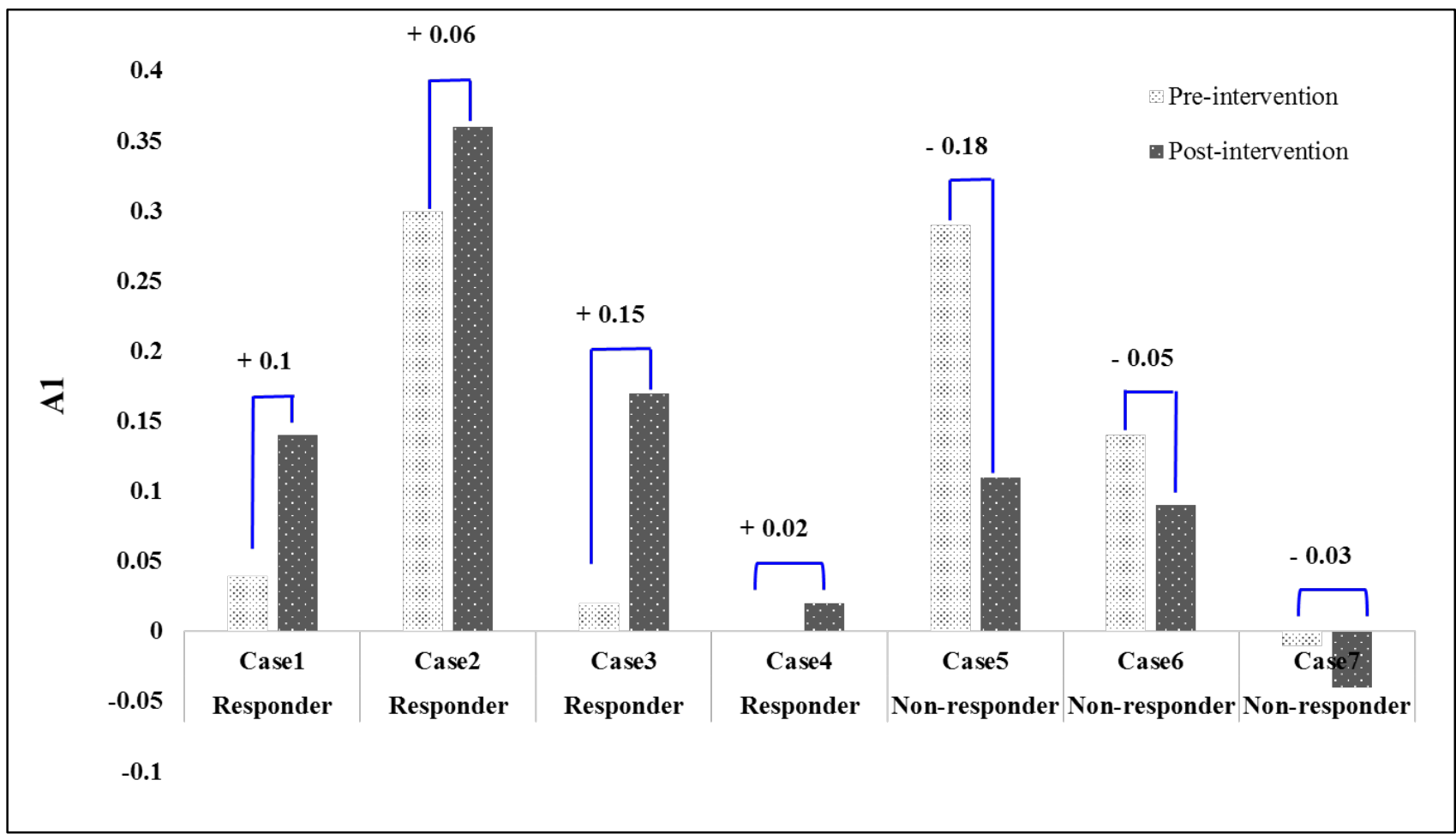

Figure 1. The A1 score at pre- and post-interventions between the responders and non-responders in the NFB group. Note: Change-score of $A 1=(A 1$ at post-intervention $)-(A 1$ at pre-intervention $)$.

The responders showed a decrease in anxiety and depression scores as compared to the nonresponders in the NFB group.

In the NFB group, anxiety and depression scores were decreased slightly from pre- to postinterventions (BAI: 23.00 to 18.00 ; and BDI-II: 35.75 to 29.25 , respectively) in the responder group ( $t=$ $0.99, p>0.05$; and $t=1.32, p>0.05$ ); otherwise, anxiety and depression scores were increased slightly from pre- to post-interventions (BAl: 11.00 to 24.67; and BDI-II: 22.67 to 28.33, respectively) in the non-responder group $(t=-1.39, p>0.05$; and $t=$ $-0.87, p>0.05$; Figure 2).
In addition, we used similar analysis of the responder group and non-responder group for the control group. The results showed that in the control group, the scores of anxiety and depression decreased slightly from pre- to post-interventions in the responder group (BAl: 15.00 to 10.75; and BDIII: 19.00 to 13.75 , respectively); otherwise, anxiety and depression scores were increased or did not change from pre- to post-interventions (BAI: 17.33 to 19.33; and BDI-II: 28.00 to 28.00 , respectively) in the non-responder group (Figure 2). 


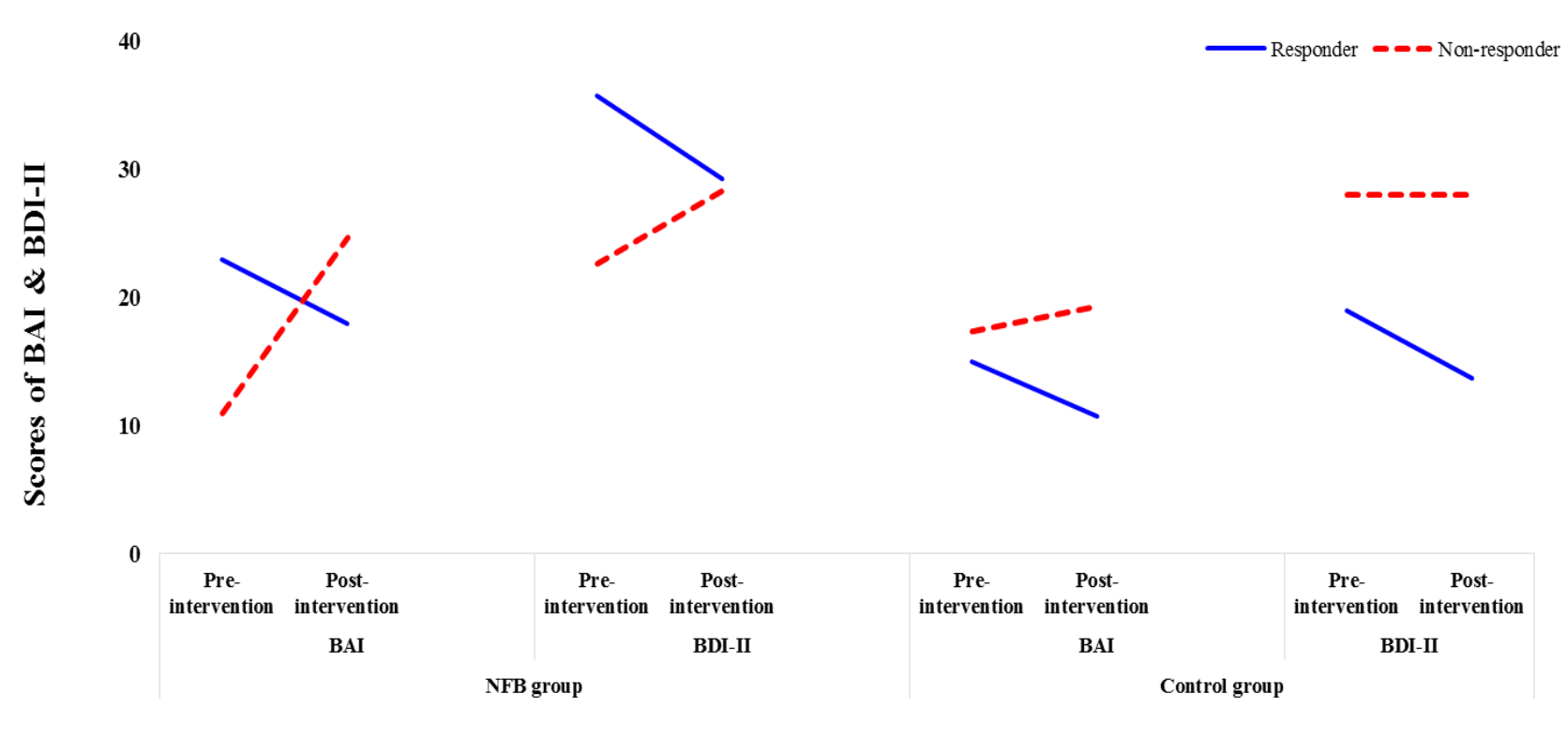

Figure 2. The scores of BAI and BDI-II at pre- and post-interventions between the responders and non-responders for the NFB and control groups.

\section{Discussion}

Preliminary results show that patients with MDD showed increased A1 score after neurofeedback training in the NFB group. The responder group responded to the neurofeedback with more improvement in anxiety and depression than the non-responder group. By separating the responders and non-responders, the study points out that it is not the number of training sessions that is important, but whether the skill mastery has been achieved. Clinical conclusions should only be drawn from those participants who mastered the skill. These results suggest that six training sessions were either insufficient for the non-responders and they needed more sessions to develop mastery or that the training protocol was inappropriate and they needed a different type of intervention (Shellenberger \& Green, 1986).

This study indicated that neurofeedback training increases the A1 score by improving frontal alpha asymmetry because of two reasons: (1) increased right frontal alpha power means decreased right frontal activity, and (2) decreased left frontal alpha power means increased left frontal activity. This result was consistent with previous studies that showed decreased depressive symptoms after neurofeedback training (Baehr et al., 1997; Baehr et al., 2001; Choi et al., 2011; Dias et al., 2011; Peeters et al., 2014; Rosenfeld, 2000). We found that patients with MDD with increased A1 score after neurofeedback training (responder) also showed decreased anxiety and depression; on the other hand, patients with MDD with decreased A1 score after neurofeedback training (non-responder) showed increased anxiety and depression.

However, this study found that not all patients with MDD increased A1 score after neurofeedback training. Even though the ALAY protocol of neurofeedback was followed to address frontal alpha asymmetry, not all patients with MDD had A1 score lower than zero. Some patients with MDD had A1 score higher than zero. Overall, this study found that increased A1 score was related to decreased symptoms of anxiety and depression $(r=-0.27)$. However, this result may due to the placebo effect and needs to be reassessed in the same experimental conditions.

Depressive disorder is a heteroscedastic disorder, the pathophysiological mechanisms of which are still controversial. Some depressive disorders are comorbid with anxiety disorder or anxiety symptoms (Bruder et al., 1997), with melancholia or nonmelancholia (Quinn, Rennie, Harris, \& Kemp, 2014), and psychomotor retardation (Cantisani et al., 2015). These heteroscedastic characteristics may reflect different EEG patterns in different brain regions. For example, patients with MDD with comorbid anxiety had higher activity in the right parietal-temporal lobe than those with MDD without anxiety (Bruder et al., 1997). 
Several limitations should be noted in this study. First, only 14 patients with MDD were included in this present study. The insufficient sample size may decrease the statistical power and cause no significant finding. Second, the neurofeedback protocol for patients with MDD needed at least 10 36 sessions in the previous studies (Baehr et al., 1997; Choi et al., 2011; Peeters et al., 2014; Rosenfeld et al., 1996); however, our participants in the NFB group only received $1 \mathrm{hr}$ per week for 6 weeks of neurofeedback training which may limit the efficacy of neurofeedback. More practice may improve neuroplasticity (Malkowicz \& Martinez, 2009). Third, the pathophysiological mechanism of alpha asymmetry was not confirmed for all patients with MDD, even though there was higher alpha asymmetry score among patients with MDD than in the healthy controls (Debener et al., 2000; Kemp et al., 2010). Fourth, because of the heterogeneity of depressive disorders, some patients with MDD with comorbid mild anxiety symptoms in our study showed a BAI score of 17.86 in the NFB group and 16.00 in the control group. Previous studies found hyperactivity in the right posterior regions in patients with comorbid MDD and anxiety symptoms (Bruder et al., 1997; Heller, Etienne, \& Miller, 1995), and the pathophysiological mechanism of EEG patterns may differ between MDD with anxiety and MDD without anxiety (Bruder et al., 1997). Therefore, the individualized neurofeedback protocol should be set up for these patients. Fifth, depressive symptoms in some patients were caused by stressful life events or adjustment problems and some patients with MDD improved significantly after few sessions of neurofeedback; however, some of them showed increased severity of depression during neurofeedback training. Hammond (2005) indicated that not all individuals with frontal alpha asymmetry will be depressed, and some persons can experience negative life events and still become depressed in the absence of frontal alpha asymmetry. Some patients did not improve significantly because of other stressful life events during neurofeedback training, such as a loss in their family (Hammond, 2005). Therefore, stressful life events may be a confounding factor in the outcome evaluation. Sixth, some studies examined the learning curve across and within neurofeedback sessions (Baehr et al., 1997; Zuberer, Brandeis, \& Drechsler, 2015) to confirm a linear trend of EEG changes. However, this study did not measure the scores of frontal alpha asymmetry under each session, and hence, the trend of treatment effectiveness is still unknown.
In conclusion, there was partial support for the efficacy of neurofeedback among patients with MDD, especially for those who were responders. Patients in the responder group showed decreased symptoms of anxiety and depression, as well as improved frontal alpha asymmetry. However, some of them did not improve significantly and probably needed more training sessions. Finally, the sample size should be increased in future studies.

\section{Author Note}

This research was supported by Kaohsiung Medical University, Taiwan (grant number KMU-Q100001).

\section{References}

American Psychiatric Association. (2013). Diagnostic and Statistical Manual of Mental Disorders (5th ed.). Washington, DC: Author.

Baehr, E., Rosenfeld, J. P., \& Baehr, R. (1997). The clinical use of an alpha asymmetry protocol in the neurofeedback treatment of depression: Two case studies. Journal of Neurotherapy, 2(3), 10-23. http://dx.doi.org/10.1300/J184v02n03 02

Baehr, E., Rosenfeld, J. P., \& Baehr, R. (2001). Clinical use of an alpha asymmetry neurofeedback protocol in the treatment of mood disorders: Follow-up study one to five years post therapy. Journal of Neurotherapy, 4(4), 11-18. http://dx.doi.org/10.1300/J184v04n04_03

Baehr, E., Rosenfeld, J. P., Baehr, R., \& Earnest, C. (1998). Comparison of two EEG asymmetry indices in depressed patients vs. normal controls. International Journal of Psychophysiology, 31(1), 89-92. http://dx.doi.org/10.1016 IS0167-8760(98)00041-5

BioGraph Infiniti (Version 6.1.1) [Computer software]. Montreal, QC, Canada: Thought Technology Ltd.

BrainAvatar (Version 4.0) [Computer software]. Bedford, OH: BrainMaster Technologies, Inc.

Bruder, G. E., Fong, R., Tenke, C. E., Leite, P., Towey, J. P., Stewart, J. E., ... Quitkin, F. M. (1997). Regional brain asymmetries in major depression with or without an anxiety disorder: A quantitative electroencephalographic study. Biological Psychiatry, 41(9), 939-948. http://dx.doi.org/10.1016 /S0006-3223(96)00260-0

Cantisani, A., Koenig, T., Horn, H., Müller, T., Strik, W., \& Walther, S. (2015). Psychomotor retardation is linked to frontal alpha asymmetry in major depression. Journal of Affective Disorders, 188, 167-172. http://dx.doi.org/10.1016 /j.jad.2015.08.018

Choi, S. W., Chi, S. E., Chung, S. Y., Kim, J. W., Ahn, C. Y., \& $\mathrm{Kim}, \mathrm{H}$. T. (2011). Is alpha wave neurofeedback effective with randomized clinical trials in depression? A pilot study. Neuropsychobiology, 63(1), 43-51. http://dx.doi.org/10.1159 1000322290

Coan, J. A., \& Allen, J. J. B. (2004). Frontal EEG asymmetry as a moderator and mediator of emotion. Biological Psychology, 67(1-2), 7-50. /j.biopsycho.2004.03.002

Davidson, R. J. (1984). Affect, cognition, and hemispheric specialization. In C. E. Izard, J. Kagan, \& R. B. Zajonc (Eds.), Emotions, Cognition, and Behavior (pp. 320-365). New York: Cambridge University Press.

Davidson, R. J. (1993). Cerebral asymmetry and emotion: Conceptual and methodological conundrums. Cognition and Emotion, 7(1), 115-138. http://dx.doi.org/10.1080 /02699939308409180 
Davidson, R. J. (1998). Anterior electrophysiological asymmetries, emotion, and depression: Conceptual and methodological conundrums. Psychophysiology, 35(5), 607-614. http://dx.doi.org/10.1017/S0048577298000134

Debener, S., Beauducel, A., Nessler, D., Brocke, B., Heilemann, H., \& Kayser, J. (2000). Is resting anterior EEG alpha asymmetry a trait marker for depression? Neuropsychobiology, 41(1), 31-37. http://dx.doi.org/10.1159 1000026630

Delorme, A., \& Makeig, S. (2004). EEGLAB: An open source toolbox for analysis of single-trial EEG dynamics including independent component analysis. Journal of Neuroscience Methods, 134(1), 9-21. http://dx.doi.org/10.1016 /j.jneumeth.2003.10.009

Dias, Á. M., \& van Deusen, A. (2011). A new neurofeedback protocol for depression. The Spanish Journal of Psychology, 14(1), 374-384. $\quad$ http://dx.doi.org/10.5209 /rev_SJOP.2011.v14.n1.34

Ebrahim, S., Bance, S., Athale, A., Malachowski, C., \& loannidis J. P. A. (2016). Meta-analyses with industry involvement are massively published and report no caveats for antidepressants. Journal of Clinical Epidemiology, 70, 155163. http://dx.doi.org/10.1016/j.jclinepi.2015.08.021

Gotlib, I. H., Ranganath, C., \& Rosenfeld, J. P. (1998). Frontal EEG alpha asymmetry, depression, and cognitive functioning. Cognition and Emotion, 12(3), 449-478. http://dx.doi.org /10.1080/026999398379673

Hammond, D. C. (2005). Neurofeedback treatment of depression and anxiety. Journal of Adult Development, 12(2), 131-137. http://dx.doi.org/10.1007/s10804-005-7029-5

Heller, W., Etienne, M. A., \& Miller, G. A. (1995). Patterns of perceptual asymmetry in depression and anxiety: Implications for neuropsychological models of emotion and psychopathology. Journal of Abnormal Psychology, 104(2), 327-333. http://dx.doi.org/10.1037/0021-843X.104.2.327

Kemp, A. H., Griffiths, K., Felmingham, K. L., Shankman, S. A., Drinkenburg, W., Arns, M., ... Bryant, R. A. (2010). Disorder specificity despite comorbidity: Resting EEG alpha asymmetry in major depressive disorder and post-traumatic stress disorder. Biological Psychology, 85(2), 350-354. http://dx.doi.org/10.1016/j.biopsycho.2010.08.001

Le Noury, J., Nardo, J. M., Healy, D., Jureidini, J., Raven, M., Tufanaru, C., \& Abi-Jaoude, E. (2015). Restoring Study 329: Efficacy and harms of paroxetine and imipramine in treatment of major depression in adolescence. BMJ, 351(h4320), 1-16. http://dx.doi.org/10.1136/bmj.h4320

Linden, M., Habib, T., \& Radojevic, V. (1996). A controlled study of the effects of EEG biofeedback on cognition and behavior of children with attention deficit disorder and learning disabilities. Biofeedback and Self-Regulation, 21(1), 35-49.

Malkowicz, D., \& Martinez, D. (2009). Role of quantitative electroencephalography, neurotherapy, and neuroplasticity in recovery from neurological and psychiatric disorders. Journal of Neurotherapy, 13(3), 176-188. http://dx.doi.org/10.1080 /10874200903127049

MATLAB (Release 2008a) [Computer software]. (2008). Natick, MA: The Math Works, Inc. Retrieved from: http://www.mathworks.com/products/new_products/release20 08a.html

Peeters, F., Oehlen, M., Ronner, J., van Os, J., \& Lousberg, R. (2014). Neurofeedback as a treatment for major depressive disorder - A pilot study. PloS ONE, 9(3), e91837. http://dx.doi.org/10.1371/journal.pone.0091837

Quinn, C. R., Rennie, C. J., Harris, A. W. F., \& Kemp, A. H. (2014). The impact of melancholia versus non-melancholia on resting-state, EEG alpha asymmetry: Electrophysiological evidence for depression heterogeneity. Psychiatry Research, 215(3), 614-617. http://dx.doi.org/10.1016 /j.psychres.2013.12.049

Rosenfeld, J. P. (2000). An EEG biofeedback protocol for affective disorders. Clinical EEG and Neuroscience, 31(1), 712. http://dx.doi.org/10.1177/155005940003100106

Rosenfeld, J. P., Baehr, E., Baehr, R., Gotlib, I. H., \& Ranganath, C. (1996). Preliminary evidence that daily changes in frontal alpha asymmetry correlate with changes in affect in therapy sessions. International Journal of Psychophysiology, 23(1-2), 137-141. http://dx.doi.org/10.1016/0167-8760(96)00037-2

Shellenberger, R., \& Green, J. A. (1986). From the ghost in the box to successful biofeedback training. Greeley, CO: Health Psychology Publications.

Stewart, J. L., Coan, J. A., Towers, D. N., \& Allen, J. J. B. (2011) Frontal EEG asymmetry during emotional challenge differentiates individuals with and without lifetime major depressive disorder. Journal of Affective Disorders, 129(1-3), 167-174. http://dx.doi.org/10.1016/j.jad.2010.08.029

Zuberer, A., Brandeis, D., \& Drechsler, R. (2015). Are treatment effects of neurofeedback training in children with ADHD related to the successful regulation of brain activity? A review on the learning of regulation of brain activity and a contribution to the discussion on specificity. Frontiers in Human Neuroscience, 9(135), 1-15. http://dx.doi.org /10.3389/fnhum.2015.00135

Received: July 12, 2016

Accepted: August 6, 2016

Published: September 2, 2016 